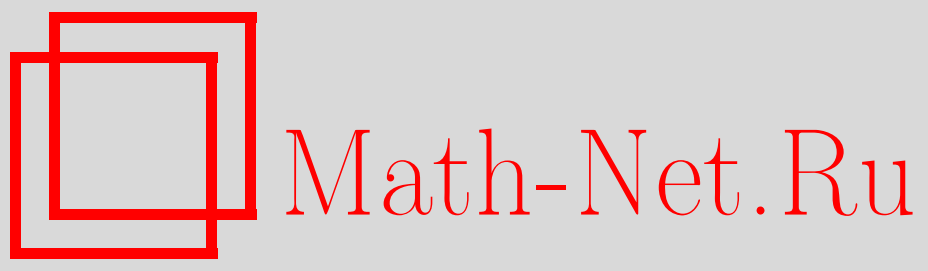

О. А. Кривошеева, А. С. Кривошеев, Особые точки суммы ряда Дирихле на прямой сходимости, Функи. анализ и его прил., 2015, том 49, выпуск $2,54-69$

DOI: https://doi.org/10.4213/faa3186

Использование Общероссийского математического портала MathNet.Ru подразумевает, что вы прочитали и согласны с пользовательским соглашением

http://www. mathnet.ru/rus/agreement

Параметры загрузки:

IP : 35.173 .137 .237

26 апреля 2023 г., 16:54:44

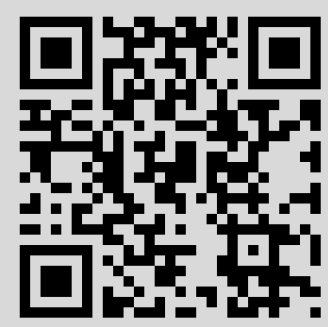


Функционалъный анализ и его приложения

2015, т. 49, вып. 2, с. 54-69

УДК 517.537.7

\title{
Особые точки суммы ряда Дирихле на прямой сходимости
}

\author{
(c) 2015. О. А. КривошеевА, А. С. Кривошеев
}

В работе исследуется проблема распределения особых точек суммы ряда Дирихле. Получены необходимые и достаточные условия, при которых каждая сумма такого ряда имеет по крайней мере одну особую точку на любом отрезке фиксированной длины, лежащем на прямой сходимости.

\section{§1. Введение}

Пусть $\Lambda=\left\{\lambda_{k}\right\}_{k=1}^{\infty}-$ неограниченная строго возрастающая последовательность положительных чисел и $n(r, \Lambda)$ - число ее членов, попавших в полуинтервал $(0, r]$. Говорят, что $\Lambda$ имеет плотность $\tau(\Lambda)$ (измерима), если существует предел

$$
\tau(\Lambda)=\lim _{r \rightarrow \infty} \frac{n(r, \Lambda)}{r} .
$$

В работе рассматриваются ряды Дирихле

$$
\sum_{k=1}^{\infty} d_{k} \exp \left(\lambda_{k} z\right)
$$

Известно (см., например, [1, гл. II, §1, п. 4]), что при некотором естественном условии на показатели $\lambda_{k}$ ряд (1) сходится абсолютно и равномерно на компактах в полуплоскости $\{z \in \mathbb{C}: \operatorname{Re} z<\gamma\}$ к аналитической функции и расходится в полуплоскости $\{z \in \mathbb{C}: \operatorname{Re} z>\gamma\}$. Число $\gamma$, называемое абсциссой сходимости, вычисляется по формуле, которая является аналогом формулы Коши-Адамара для степенных рядов (см., например, [1, гл. II, §1, п. 4, теорема 2.1.2]). Отметим, кроме того, что разложение функций в ряд Дирихле всегда является единственным (см., например, [1, гл. II, §1, п. 3]).

Цель работы - исследование проблемы распределения особых точек суммы ряда (1) на прямой сходимости $L(\gamma)=\{z \in \mathbb{C}: \operatorname{Re} z=\gamma\}$. Первые результаты в этом направлении были получены еще в позапрошлом веке. Они были связаны с частным случаем рядов (1) - степенными рядами

$$
\sum_{k=1}^{\infty} d_{k} w^{\lambda_{k}}, \quad \lambda_{k} \in \mathbb{N},
$$

которые при замене $w=\exp z$ переходят в ряды Дирихле. В 1892 г. Адамар [2] доказал, что если функция $g$ представляется рядом (2) с пропусками $\lambda_{k+1}-\lambda_{k} \geqslant \alpha \lambda_{k}, k=1,2, \ldots$, где $\alpha$ - положительное число, то граница круга сходимости этого ряда является естественной границей области существования функции $g$, т. е. каждая точка этой границы является особой для $g$. Фабри [3] в 
1896 г. доказал, что утверждение Адамара сохраняется при более общем условии на последовательность показателей, а именно, $\Lambda$ должна иметь нулевую плотность $\tau(\Lambda)$. Существенное обобщение этого результата получил Пойа. При этом важную роль сыграло введенное им понятие максимальной плотности последовательности положительных чисел

$$
\tau_{0}(\Lambda)=\varlimsup_{\delta \rightarrow 0} \varlimsup_{r \rightarrow \infty} \frac{n(r, \Lambda)-n((1-\delta) r, \Lambda)}{\delta r} .
$$

Отметим, что, согласно лемме из разд. Е3 гл. VI книги [4], верхний предел при $\delta \rightarrow 0$ в определении плотности $\tau_{0}(\Lambda)$ можно заменить на предел (т. е. предел всегда существует). Пойа [5] показал, что при условии, что $\tau_{0}(\Lambda)<\infty$, сумма ряда (2), сходящегося в единичном круге, на каждой дуге единичной окружности длины $2 \pi \tau_{0}(\Lambda)$ имеет хотя бы одну особую точку. Этому результату предшествовали тонкие результаты Фабри об особых точках суммы степенного ряда на дугах окружности сходимости (см. [6, §2]). Позднее Фукс [7] и Мальявен [8] получили результат, обратный к теореме Пойи. Для каждой последовательности $\Lambda \subset \mathbb{N}$, удовлетворяющей условию $\tau_{0}(\Lambda)<\infty$, и каждого $\varepsilon>0$ был построен ряд (2), сходящийся в единичном круге, сумма которого аналитически продолжается через дугу единичной окружности $\left\{e^{i \varphi}:|\varphi|<\pi \tau_{0}(\Lambda)-\varepsilon\right\}$. Полное доказательство этого очень сложного результата, основанного на конструкции Фукса, приведено в гл. IX книги [9].

Параллельно с исследованиями степенных рядов изучались и более общие ряды Дирихле. Пойа ([10], [11]), а также Карлсон и Ландау (см., например, [1, гл II, §5.2]) распространили результат Фабри на случай рядов Дирихле. Они показали, что для ряда (1), последовательность $\Lambda$ показателей которого имеет нулевую плотность и удовлетворяет условию $\lambda_{k+1}-\lambda_{k} \geqslant h>0, k=1,2, \ldots$, справедливо следующее утверждение: либо его сумма есть целая функция, либо прямая сходимости является естественной границей области существования этой суммы. Этот результат является частным случаем более общего утверждения Бернштейна [12]. Сформулируем его. Пусть последовательность $\Lambda$ имеет конечную плотность $\tau(\Lambda)$. Положим

$$
L(\lambda)=\prod_{k=1}^{\infty}\left(1-\frac{\lambda^{2}}{\lambda_{k}^{2}}\right)
$$

Индексом конденсации Бернштейна называется величина, определяемая формулой

$$
\gamma(\Lambda)=\varlimsup_{k \rightarrow \infty} \frac{1}{\lambda_{k}} \ln \left|\frac{1}{L^{\prime}\left(\lambda_{k}\right)}\right| .
$$

Бернштейн доказал, что при условии $\gamma(\Lambda)=0$ каждый отрезок длины $2 \pi \tau(\Lambda)$ прямой сходимости ряда (1) (если таковая имеется) содержит по крайней мере одну особую точку его суммы. Отметим, что это утверждение ранее доказал Пойа [10] при более сильном ограничении на последовательность $\Lambda=\left\{\lambda_{k}\right\}$ : $\lambda_{k+1}-\lambda_{k} \geqslant h>0, k=1,2, \ldots$ В книге [13, гл. II, §3.3] строится специальная функция, которая является суммой ряда Дирихле и при условии $\gamma(\Lambda) \neq 0$ не имеет особых точек на прямой сходимости. Существование такой функции говорит о том, что условие $\gamma(\Lambda)=0$ в теореме Бернштейна необходимо.

В работе [14] исследовалась проблема распределения особых точек суммы ряда экспонент (и более общих рядов экспоненциальных мономов) на границе его 
области сходимости. Был доказан ряд общих утверждений, следствиями которых являются большинство отмеченных здесь результатов. При этом важную роль сыграла характеристика $S_{\Lambda}$ последовательности $\Lambda$, введенная в работе [15] (ее мы определим ниже), которая является аналогом индекса конденсации Бернштейна-Леонтьева (индекса конденсации Бернштейна, распространенного на ряды экспонент). В отличие от последнего $(\gamma(\Lambda)$ используется для измеримых последовательностей) он эффективен для произвольных комплексных последовательностей.

В данной работе на основе исследований из работы [16] для рядов Дирихле получен результат, который является аналогом теоремы Пойи для степенных рядов. Кроме того, получено обратное утверждение, которое является аналогом теоремы Фукса и Мальявена. Его доказательство опирается на полное решение проблемы фундаментального принципа для инвариантных относительно оператора дифференцирования подпространств, найденное в работах [15] и [16]. Оба указанных результата сформулированы в виде одной теоремы.

Теорема 1. Пусть $\Lambda=\left\{\lambda_{k}\right\}_{k=1}^{\infty}-$ неограниченная строго возрастающая последовательность положительных чисел и $\tau>0$. Следующие утверждения эквивалентны.

1. Каждая сумма ряда (1), не являющаяся целой функцией, на каждом отрезке длины $2 \pi \tau$ прямой сходимости имеет хотя бы одну особую точку.

2. $S_{\Lambda}=0 u \tau_{0}(\Lambda) \leqslant \tau$.

Замечание. Точка $z$ прямой сходимости ряда (1) называется особой для его суммы, если не существует функции, аналитической в объединении полуплоскости сходимости и какого-либо круга с центром в $z$, совпадающей с суммой этого ряда в этой полуплоскости.

Доказательству теоремы 1 посвящен $\S 3$ работы. В $\S 2$ собраны необходимые для этого вспомогательные утверждения.

\section{§2. Предварительные результаты}

Чтобы доказать теорему 1, нам необходимо адаптировать результаты работ [14] и [15] к рассматриваемой здесь ситуации. Для этого мы напомним некоторые известные определения и докажем ряд вспомогательных утверждений.

Пусть $\Lambda=\left\{\lambda_{k}\right\}_{k=1}^{\infty}-$ такая же последовательность, как в теореме 1 . Положим

$$
\bar{\tau}(\Lambda)=\varlimsup_{r \rightarrow \infty} \frac{n(r, \Lambda)}{r} .
$$

Величина $\bar{\tau}(\Lambda)$ называется верхней плотностью последовательности $\Lambda$. Нетрудно заметить, что

$$
\bar{\tau}(\Lambda) \leqslant \tau_{0}(\Lambda) .
$$

Действительно, если $\bar{\tau}(\Lambda)<+\infty$, то

$$
\begin{aligned}
\varlimsup_{r \rightarrow \infty} \frac{n(r, \Lambda)-n((1-\delta) r, \Lambda)}{\delta r} & \geqslant \varlimsup_{r \rightarrow \infty} \frac{n(r, \Lambda)}{\delta r}-\varlimsup_{r \rightarrow \infty} \frac{n((1-\delta) r, \Lambda)}{\delta r} \\
& =\frac{\bar{\tau}(\Lambda)}{\delta}-(1-\delta) \frac{\bar{\tau}(\Lambda)}{\delta}=\bar{\tau}(\Lambda), \quad \delta>0 .
\end{aligned}
$$

Если же $\bar{\tau}(\Lambda)=+\infty$, то из последовательности $\Lambda$ нетрудно выделить подпоследовательность $\Lambda^{\prime}$ со сколь угодно большой конечной верхней плотностью. Тогда 
по доказанному получаем неравенства $\bar{\tau}\left(\Lambda^{\prime}\right) \leqslant \tau_{0}\left(\Lambda^{\prime}\right) \leqslant \tau_{0}(\Lambda)$. Следовательно, $\tau_{0}(\Lambda)=+\infty$, т. е. неравенство $(3)$ верно и в этом случае. Схожие выкладки показывают, что если последовательность имеет плотность $\tau(\Lambda)$, то $\tau_{0}(\Lambda)=\tau(\Lambda)$. В общем случае неравенство (3) может быть строгим. Действительно, рассмотрим следующий пример. Пусть $\Lambda=\bigcup_{m=1}^{\infty} \Lambda_{m}$, где $\Lambda_{m}=\left\{\lambda_{k}, k(m) \leqslant k<\right.$ $k(m+1)\}, \lambda_{k(m)+j}=10^{m}+j, 0 \leqslant j<k(m+1)-k(m), m=1,2, \ldots$, и $k(1)=1$, $k(m+1)-k(m)=10^{m-1}$ при $m>1$. Непосредственным подсчетом нетрудно получить соотношения $\bar{\tau}(\Lambda) \leqslant 1 / 9, \tau_{0}(\Lambda)=1$.

Пусть $\Lambda=\left\{\lambda_{k}\right\}_{k=1}^{\infty}, \tilde{\Lambda}=\left\{\mu_{n}\right\}_{n=1}^{\infty}$. Будем говорить, что $\Lambda$ является частью последовательности $\tilde{\Lambda}(\Lambda \subset \tilde{\Lambda})$ или $\tilde{\Lambda}$ является пополнением последовательности $\Lambda$, если существует подпоследовательность $\left\{\mu_{n(k)}\right\}$, совпадающая с $\left\{\lambda_{k}\right\}$. Известная теорема Пойи (см., например, [4, гл. VI, §Е3]) утверждает, что любая последовательность с конечной максимальной плотностью является частью некоторой измеримой последовательности с той же плотностью.

Нам понадобятся некоторые сведения из теории целых функций экспоненциального типа, т. е. функций $f$, удовлетворяющих оценке $\ln |f(z)| \leqslant A+B|z|$, $z \in \mathbb{C}$, где $A, B>0$ зависят от $f$. Верхним и нижним индикаторами функции $f$ называются соответственно функции

$$
h_{f}(\lambda)=\varlimsup_{t \rightarrow \infty} \frac{\ln |f(t \lambda)|}{t}, \quad \underline{h}_{f}(\lambda)=\lim _{\delta \rightarrow 0} \frac{\lim }{t \rightarrow \infty} \frac{1}{\pi \delta^{2}} \int_{B(\lambda, \delta)} \frac{\ln |f(t z)|}{t} d x d y, \quad \lambda \in \mathbb{C},
$$

где $z=x+i y$. Из этих определений и теоремы Хартогса о верхнем пределе семейства субгармонических функций нетрудно получить неравенство $\underline{h}_{f}(\lambda) \leqslant$ $h_{f}(\lambda), \lambda \in \mathbb{C}$. Говорят (см. [17, гл. III]), что $f$ имеет (вполне) регулярный рост, если

$$
h_{f}(\lambda)=\lim _{t \rightarrow \infty, t \notin E} \frac{\ln |f(t \lambda)|}{t}, \quad \lambda \in \mathbb{C},
$$

где $E-$ множество нулевой относительной меры на луче $(0,+\infty)$, т. е. мера Лебега его пересечения с интервалом $(0, r)$ бесконечна мала по сравнению с $r$ при $r \rightarrow+\infty$. Имеется ряд других эквивалентных этому определений регулярности роста. Приведем одно из них. Функция $f$ называется (см. [18, гл. 4, определение 4.1]) функцией регулярного роста, если $\underline{h}_{f}(\lambda)=h_{f}(\lambda), \lambda \in \mathbb{C}$.

Верхний индикатор $h_{f}$ является выпуклой положительно однородной порядка один функцией, которая совпадает с опорной функцией выпуклого компакта $K$, называемого сопряженной диаграммой функции $f$ (см., например, [13, гл. I, $\S 5$, теорема 5.4 (Пойа)]):

$$
h_{f}(\lambda)=H_{K}(\lambda)=\sup _{z \in K} \operatorname{Re}(\lambda z), \quad \lambda \in \mathbb{C} .
$$

Пусть $\Lambda=\left\{\lambda_{k}\right\}_{k=1}^{\infty}-$ последовательность комплексных чисел с единственной предельной точкой $\infty$. Символом $n\left(\varphi_{1}, \varphi_{2}, r, \Lambda\right)$ обозначим число точек $\lambda_{k}$, попавших в сектор $\left\{\lambda=t e^{i \varphi}: \varphi \in\left(\varphi_{1}, \varphi_{2}\right), t \in(0, r)\right\}$. Говорят (см. [17, гл. II, §1]), что $\Lambda$ имеет угловую плотность (при порядке один), если для всех значений $\varphi_{1}$, $\varphi_{2}$, за исключением, быть может, счетного множества, существует предел

$$
\tau\left(\varphi_{1}, \varphi_{2}, \Lambda\right)=\lim _{r \rightarrow \infty} \frac{n\left(\varphi_{1}, \varphi_{2}, r, \Lambda\right)}{r} .
$$


Множество $\Lambda$ называется правильно распределенным, если оно имеет угловую плотность и существует

$$
\lim _{r \rightarrow \infty} \sum_{\left|\lambda_{k}\right|<r} \frac{1}{\lambda_{k}} .
$$

Согласно теореме 4 гл. III книги [17], функция $f$ имеет регулярный рост тогда и только тогда, когда ее нулевое множество (с учетом кратностей) является правильно распределенным. При этом, если $K$ - сопряженная диаграмма функции $f$, то для всех, за исключением, быть может, счетного числа, значений $\varphi_{1}, \varphi_{2}$ верно равенство (см. [17, гл. II, §1, формула (2.07)])

$$
\tau\left(\varphi_{1}, \varphi_{2}, \Lambda\right)=\frac{1}{2 \pi} s\left(\varphi_{1}, \varphi_{2}, K\right),
$$

где $s\left(\varphi_{1}, \varphi_{2}, K\right)$ - длина дуги границы $\partial K$, заключенной между точками опоры $z\left(\varphi_{1}\right) \in \partial K$ и $z\left(\varphi_{2}\right) \in \partial K$ опорных прямых $l\left(\varphi_{1}\right)=\left\{z: \operatorname{Re}\left(z e^{i \varphi_{1}}\right)=H_{K}\left(e^{i \varphi_{1}}\right)\right\}$ и $l\left(\varphi_{2}\right)=\left\{z: \operatorname{Re}\left(z e^{i \varphi_{2}}\right)=H_{K}\left(e^{i \varphi_{2}}\right)\right\}$ соответственно. Для всех значений $\varphi$, за исключением не более чем счетного числа значений (соответствующих прямолинейным участкам границы), опорная прямая $l(\varphi)$ имеет единственную точку опоры $z(\varphi)$. Из двух дуг, соединяющих точки $z\left(\varphi_{1}\right)$ и $z\left(\varphi_{2}\right)$, выбирается та, каждая точка которой является точкой опоры некоторой прямой $l(\varphi)$ со значением параметра $\varphi$ из отрезка $\left[\varphi_{1}, \varphi_{2}\right]$.

Лемма 1. Пусть $\Lambda=\left\{\lambda_{k}\right\}_{k=1}^{\infty}-$ неограниченная строго возрастаящая последовательность положительных чисел с конечной максимальной плотностью $\tau_{0}(\Lambda)$. Тогда существует целая функиия $f$ экспоненциального типа и регулярного роста, обращающаяся в нуль в точках $\lambda_{k}, k \geqslant 1$, сопряженная диаграмма которой является отрезком мнимой оси $\left[-i \pi \tau_{0}, i \pi \tau_{0}\right]$.

Доказательство. По указанной выше теореме Пойи существует пополнение $\tilde{\Lambda}=\left\{\mu_{n}\right\}_{n=1}^{\infty}$ последовательности $\Lambda$, такое, что $\tau(\tilde{\Lambda})=\tau_{0}(\Lambda)$. Рассмотрим функцию

$$
f(\lambda)=\prod_{n=1}^{\infty}\left(1-\frac{\lambda^{2}}{\mu_{n}^{2}}\right) .
$$

Она обращается в нуль в точках $\lambda_{k}, k \geqslant 1$. Поскольку $\tilde{\Lambda}$ измерима, то легко проверить, что нулевое множество функции $f(\lambda)$ является правильно распределенным. Поэтому $f$ является целой функцией экспоненциального типа и регулярного роста. По теореме 1.2 .9 из книги $[1$, гл. I, §2] сопряженная диаграмма функции $f$ совпадает с отрезком мнимой оси $\left[-i \pi \tau_{0}(\Lambda), i \pi \tau_{0}(\Lambda)\right]$. Лемма доказана.

Пусть $\Lambda=\left\{\lambda_{k}\right\}_{k=1}^{\infty}-$ последовательность положительных чисел. Следуя работе [15], положим

$$
q_{\Lambda}^{j}(z, \delta)=\prod_{\lambda_{k} \in B\left(\lambda_{j}, \delta \lambda_{j}\right), k \neq j} \frac{z-\lambda_{k}}{3 \delta \lambda_{k}} .
$$

Здесь $B(w, r)$ - открытый круг с центром в $w$ и радиуса $r$. Модуль функции $q_{\Lambda}^{j}(z, \delta)$ можно интерпретировать как меру сгущения точек $\lambda_{k} \in B\left(\lambda_{j}, \delta \lambda_{j}\right), k \neq j$, около $z$. В случае, когда такие точки отсутствуют, считаем, что $q_{\Lambda}^{j}(z, \delta) \equiv 1$. Отметим, что модуль каждого из сомножителей в определении функции $q_{\Lambda}^{j}(z, \delta)$ 
в круге $B\left(\lambda_{j}, \delta \lambda_{j}\right)$ оценивается сверху величиной $2(3(1-\delta))^{-1}$ (для $\left.\delta \in(0,1)\right)$, т. е. при $\delta \in(0,1 / 3)$ он не превосходит единицы. Кроме того, если $\delta_{1} \leqslant \delta_{2}$, то число сомножителей в определении функции $q_{\Lambda}^{j}\left(z, \delta_{1}\right)$ не превосходит числа сомножителей в определении функции $q_{\Lambda}^{j}\left(z, \delta_{2}\right)$, и каждый из сомножителей для $q_{\Lambda}^{j}\left(z, \delta_{1}\right)$ по модулю не меньше соответствующего сомножителя для $q_{\Lambda}^{j}\left(z, \delta_{2}\right)$. Таким образом, если $0<\delta_{1} \leqslant \delta_{2}<1 / 3$, то

$$
\left|q_{\Lambda}^{j}\left(z, \delta_{1}\right)\right| \geqslant\left|q_{\Lambda}^{j}\left(z, \delta_{2}\right)\right|, \quad z \in \mathbb{C}
$$

Положим $S_{\Lambda}=0$, если $\Lambda$ состоит из конечного числа элементов, и

$$
S_{\Lambda}=\lim _{\delta \rightarrow 0} \underset{k \rightarrow \infty}{\lim _{k \rightarrow \infty}} \frac{\ln \left|q_{\Lambda}^{k}\left(\lambda_{k}, \delta\right)\right|}{\lambda_{k}}
$$

в противном случае. Это определение корректно, поскольку, согласно последнему неравенству, предел по $\delta$ всегда существует. В силу сказанного выше $S_{\Lambda} \leqslant 0$. Отметим, что коэффициент 3 в определении функции $q_{\Lambda}^{j}$ выбран лишь для удобства. Он обеспечивает неположительность величины $S_{\Lambda}$. Она схожа по смыслу с классическим индексом конденсации Бернштейна, но при этом эффективна для любой вещественной последовательности (не обязательно имеющей плотность). Наряду с $S_{\Lambda}$ введем еще величину

$$
\widetilde{S}_{\Lambda}=\lim _{\delta \rightarrow 0} \underset{k \rightarrow \infty}{\lim _{k \rightarrow \infty}} \frac{\ln \left|q_{\Lambda}^{k}\left(\lambda_{k}, \delta\right)\right|}{\delta \lambda_{k}} .
$$

Как и для $S_{\Lambda}$, верно неравенство $\widetilde{S}_{\Lambda} \leqslant 0$. Если $\widetilde{S}_{\Lambda}$ конечна, то, очевидно, $S_{\Lambda}=0$.

В качестве примера рассмотрим последовательность $\Lambda=\left\{\lambda_{k}\right\}_{k=1}^{\infty}$ положительных чисел, такую, что $\lambda_{k+1}-\lambda_{k} \geqslant h>0, k=1,2, \ldots$ Учитывая неравенство $n ! \geqslant(n / 3)^{n}$, имеем

$$
\begin{aligned}
\left|q_{\Lambda}^{k}\left(\lambda_{k}, \delta\right)\right| & \geqslant \prod_{\lambda_{m} \in B\left(\lambda_{k}, \delta \lambda_{k}\right), m \neq k}\left|\frac{\lambda_{m}-\lambda_{k}}{3 \delta \lambda_{m}}\right| \\
& \geqslant \frac{\left(l(k, \delta) ! h^{l(k, \delta)}\right)^{2}}{\left(3 \delta(1+\delta) \lambda_{k}\right)^{2 l(k, \delta)}} \geqslant \frac{(l(k, \delta) h)^{2 l(k, \delta)}}{\left(9 \delta(1+\delta) \lambda_{k}\right)^{2 l(k, \delta)}},
\end{aligned}
$$

где $l(k, \delta)$ - максимальное целое число, удовлетворяющее неравенству $l(k, \delta) h<$ $\delta \lambda_{k}$. Следовательно,

$$
\widetilde{S}_{\Lambda} \geqslant \frac{\lim }{\delta \rightarrow 0} \underset{k \rightarrow \infty}{\lim } \frac{2 l(k, \delta) \ln \left(l(k, \delta) h / 9 \delta(1+\delta) \lambda_{k}\right)}{\delta \lambda_{k}} \geqslant-\frac{2 \ln 9}{h}
$$

Лемма 2. Пусть $\Lambda=\left\{\lambda_{k}\right\}_{k=1}^{\infty}$ - неограниченная строго возрастающая последовательность положительных чисел. Предположим, что $\widetilde{S}_{\Lambda}>-\infty$. Тогда максимальная плотность $\tau_{0}(\Lambda)$ конечна. 
Доказательство. Пусть $\delta \in(0,1)$. Используя определение функции $q_{\Lambda}^{j}$, получаем, что

$$
\begin{aligned}
\ln \left|q_{\Lambda}^{j}\left(\lambda_{j}, \delta\right)\right| & =\ln \left|\prod_{\lambda_{k} \in B\left(\lambda_{j}, \delta \lambda_{j}\right), k \neq j} \frac{\lambda_{j}-\lambda_{k}}{3 \delta \lambda_{k}}\right| \\
& \leqslant \ln \left|\prod_{\lambda_{k} \in B\left(\lambda_{j}, \delta \lambda_{j}\right), k \neq j} \frac{\delta \lambda_{j}}{3 \delta \lambda_{k}}\right| \leqslant-(m(j, \delta)-1) \ln (3(1-\delta)),
\end{aligned}
$$

где $m(j, \delta)$ - число точек $\lambda_{k}$, попавших в круг $B\left(\lambda_{j}, \delta \lambda_{j}\right)$, т. е. число $m(j, \delta)$ равно $n\left((1+\delta) \lambda_{j}, \Lambda\right)-n\left((1-\delta) \lambda_{j}, \Lambda\right)$, если точка $(1+\delta) \lambda_{k}$ не принадлежит $\Lambda$, и на единицу меньше этой разности в противном случае. Следовательно,

$$
\begin{aligned}
\widetilde{S}_{\Lambda} & =\varliminf_{\delta \rightarrow 0} \varliminf_{k \rightarrow \infty} \frac{\ln \left|q_{\Lambda}^{k}\left(\lambda_{k}, \delta\right)\right|}{\delta \lambda_{k}} \leqslant \varliminf_{\delta \rightarrow 0} \lim _{j \rightarrow \infty} \frac{-\ln (3(1-\delta)) m(j, \delta)}{\delta \lambda_{j}} \\
& =-\ln 3 \varlimsup_{\delta \rightarrow 0} \varlimsup_{j \rightarrow \infty} \frac{n\left((1+\delta) \lambda_{j}, \Lambda\right)-n\left((1-\delta) \lambda_{j}, \Lambda\right)}{\delta \lambda_{j}} .
\end{aligned}
$$

Покажем, что двойной верхний предел в последнем равенстве оценивается снизу величиной $\tau_{0}(\Lambda)$. Если $\tau_{0}(\Lambda)=0$, то это очевидно. Для каждого $\delta \in(0,1)$ через $r_{k}(\delta), k=1,2, \ldots$, обозначим последовательность, реализующую верхний предел по $r \rightarrow \infty$ в определении максимальной плотности. Пусть $\tau_{0}(\Lambda)>0$. Тогда можно считать, что любой полуинтервал $\left((1-\delta) r_{k}(\delta), r_{k}(\delta)\right]$ содержит некоторое ненулевое число точек последовательности $\Lambda$. Произвольным образом выберем одну из них и через $j(k, \delta)$ обозначим ее номер. Поскольку $\lambda_{j(k, \delta)} \leqslant r_{k}(\delta) \leqslant$ $\lambda_{j(k, \delta)} /(1-\delta)$, то нетрудно заметить, что $\left((1-\delta) r_{k}(\delta), r_{k}(\delta)\right] \subset\left((1-\tilde{\delta}) \lambda_{j(k, \delta)}\right.$, $\left.(1+\tilde{\delta}) \lambda_{j(k, \delta)}\right]$, где $\tilde{\delta}=\delta /(1-\delta)$. Следовательно,

$$
\begin{aligned}
\tau_{0}(\Lambda) & =\varlimsup_{\delta \rightarrow 0} \lim _{j \rightarrow \infty} \frac{n\left(r_{k}(\delta), \Lambda\right)-n\left((1-\delta) r_{k}(\delta), \Lambda\right)}{\delta r_{k}(\delta)} \\
& \leqslant \varlimsup_{\delta \rightarrow 0} \varlimsup_{j \rightarrow \infty} \frac{n\left((1+\tilde{\delta}) \lambda_{j}, \Lambda\right)-n\left((1-\tilde{\delta}) \lambda_{j}, \Lambda\right)}{\tilde{\delta} \lambda_{j}} .
\end{aligned}
$$

Таким образом, с учетом предыдущего получаем, что $\widetilde{S}_{\Lambda} \leqslant-\ln 3 \tau_{0}(\Lambda)$. Отсюда следует требуемое утверждение. Лемма доказана.

Доказательство следующего утверждения основано на идеях доказательства теоремы 3.1 в работе [14].

Лемма 3. Пусть $\Lambda=\left\{\lambda_{k}\right\}_{k=1}^{\infty}-$ неограниченная строго возрастающая последовательность положительных чисел. Предположим, что $\widetilde{S}_{\Lambda}=-\infty$. Тогда для каждого $\tau>0$ существует ряд вида (1), сумма которого аналитически продолжается через некоторый отрезок длины $\tau$, лежащий на прямой сходимости этого ряда.

Доказательство. Сначала мы определим аналитическую функцию $g(z)$, а потом докажем, что она является суммой ряда Дирихле и обладает свойством, указанным в утверждении леммы. Положим

$$
g(z)=\sum_{p=1}^{\infty} c_{p} g_{p}(z),
$$


где $c_{p}=\exp \left(-6 \tau \delta \mu_{m(p)}\right)$,

$$
g_{p}(z)=\frac{1}{2 \pi i} \int_{S\left(\mu_{m(p)}, 5 \delta \mu_{m(p)}\right)} \frac{\exp (\lambda z) d \lambda}{a_{p}\left(\lambda-\mu_{m(p)}\right) q_{\tilde{\Lambda}}^{m(p)}(\lambda, \delta)}, \quad p=1,2, \ldots,
$$

$S(b, r)$ - окружность с центром в точке $b$ и радиуса $r$, а последовательность $\tilde{\Lambda}=$ $\left\{\mu_{m}\right\}_{m=1}^{\infty}$ является частью последовательности $\Lambda$. Числа $a_{p} \geqslant 1$ мы выберем ниже. Сейчас же определим число $\delta$, построим $\tilde{\Lambda}$ и подберем номера $m(p)$. Для этого, прежде всего, заметим, что, согласно условию, $\widetilde{S}_{\Lambda}=-\infty$. Поэтому найдется $\delta \in(0,1 / 4)$ и подпоследовательность $\left\{\lambda_{k(p)}\right\}_{p=1}^{\infty}$ последовательности $\Lambda$, удовлетворяющие условию

$$
\ln \left|q_{\Lambda}^{k(p)}\left(\lambda_{k(p)}, \delta\right)\right| \leqslant-6 \tau \delta \lambda_{k(p)}, \quad p=1,2, \ldots
$$

При этом можно считать, что

$$
\lambda_{k(p+1)} \geqslant 2 \lambda_{k(p)}, \quad p=1,2, \ldots
$$

Последовательность $\tilde{\Lambda} \subset \Lambda$ будем искать в виде объединения $\bigcup_{p=1}^{\infty} \Lambda_{p}$. Фиксируем $p=1,2, \ldots$ Если $n\left((1+\delta) \lambda_{k(p)}, \Lambda\right)-n\left((1-\delta) \lambda_{k(p)}, \Lambda\right)-1<12 \tau \delta \lambda_{k(p)}+1$, то в качестве $\Lambda_{p}$ возьмем множество всех точек последовательности $\Lambda$, попавших в круг $B\left(\lambda_{k(p)}, \delta \lambda_{k(p)}\right)$. В противном случае поместим в $\Lambda_{p}$ точку $\lambda_{k(p)}$ и еще столько точек из $\Lambda$, попавших в круг $B\left(\lambda_{k(p)}, \delta \lambda_{k(p)}\right)$, чтобы число точек $l(p)$ множества $\Lambda_{p}$ удовлетворяло оценкам

$$
12 \tau \delta \lambda_{k(p)} \leqslant l(p)-1<12 \tau \delta \lambda_{k(p)}+1 .
$$

Отметим, что в силу неравенства (7) и выбора числа $\delta$ круги $B\left(\lambda_{k(p)}, \delta \lambda_{k(p)}\right), p=$ $1,2, \ldots$, попарно не пересекаются. Поэтому множества $\Lambda_{p}, p=1,2, \ldots$, также попарно не пересекаются. Будем считать, что элементы $\mu_{m}$ последовательности $\tilde{\Lambda}$ пронумерованы по возрастанию. Через $m(p), p=1,2, \ldots$, обозначим номер, для которого $\mu_{m(p)}=\lambda_{k(p)}$. Имеют место неравенства

$$
\ln \left|q_{\tilde{\Lambda}}^{m(p)}\left(\mu_{m(p)}, \delta\right)\right| \leqslant-6 \tau \delta \mu_{m(p)}, \quad p=1,2, \ldots
$$

Действительно, функция $q_{\tilde{\Lambda}}^{m(p)}$ состоит из сомножителей, построенных по точкам множества $\Lambda_{p}$. Если оно совпадает с множеством точек из $\Lambda$, попавших в круг $B\left(\lambda_{k(p)}, \delta \lambda_{k(p)}\right)$, то $q_{\tilde{\Lambda}}^{m(p)}\left(\mu_{m(p)}, \delta\right)=q_{\Lambda}^{k(p)}\left(\lambda_{k(p)}, \delta\right)$ и $(9)$ вытекает из $(6)$. В противном случае, учитывая (8) и неравенство $\delta<1 / 4$, как и в лемме 2 , получаем

$$
\ln \left|q_{\tilde{\Lambda}}^{m(p)}\left(\mu_{m(p)}, \delta\right)\right| \leqslant-(l(p)-1) \ln (3(1-\delta)) \leqslant-(l(p)-1) / 2 \leqslant-6 \tau \delta \mu_{m(p)} .
$$

Покажем теперь, что верхняя плотность последовательности $\tilde{\Lambda}$ конечна. В силу (8)

$$
\frac{n\left((1+\delta) \mu_{m(p)}, \tilde{\Lambda}\right)-n\left((1-\delta) \mu_{m(p)}, \tilde{\Lambda}\right)}{\mu_{m(p)}} \leqslant 12 \tau \delta+\frac{2}{\mu_{m(p)}}, \quad p=1,2, \ldots
$$

Пусть $r>0$ и $p(r)$ - максимальный из номеров $p=1,2, \ldots$, для которых интервалы $(0, r)$ и $\left((1-\delta) \mu_{m(p)},(1+\delta) \mu_{m(p)}\right)$ пересекаются. Тогда $r>(1-\delta) \mu_{m}(p(r))$. 
Так как все точки $\mu_{m}$ лежат в объединении $\bigcup_{p=1}^{\infty}\left((1-\delta) \mu_{m(p)},(1+\delta) \mu_{m(p)}\right)$ и $\mu_{m(p)}=\lambda_{k(p)}, p=1,2, \ldots$, то с учетом (10) и (7) получаем

$$
\begin{aligned}
\frac{n(r, \tilde{\Lambda})}{r} & \leqslant \sum_{p=1}^{p(r)} \frac{n\left((1+\delta) \mu_{m(p)}, \tilde{\Lambda}\right)-n\left((1-\delta) \mu_{m(p)}, \tilde{\Lambda}\right)}{r} \\
& \leqslant \sum_{p=1}^{p(r)} \frac{12 \tau \delta \mu_{m(p)}+2}{(1-\delta) \mu_{m(p(r))}} \leqslant \frac{p(r)}{(1-\delta) 2^{p(r)-2} \mu_{m(1)}}+\sum_{p=1}^{p(r)} \frac{12 \tau \delta}{(1-\delta) 2^{p(r)-p}} .
\end{aligned}
$$

Отсюда следует, что величина $\bar{\tau}(\tilde{\Lambda})$ конечна.

Найдем оценки сверху на модули функций $g_{p}$. Так как $a_{p} \geqslant 1$, то

$$
\begin{aligned}
\left|a_{p} q_{\tilde{\Lambda}}^{m(p)}(\lambda, \delta)\right| & \geqslant\left|\prod_{\mu_{m} \in B\left(\mu_{m(p)}, \delta \mu_{m(p)}\right), m \neq m(p)} \frac{\lambda-\mu_{m}}{3 \delta \mu_{m}}\right| \\
& \geqslant \prod_{\mu_{m} \in B\left(\mu_{m(p)}, \delta \mu_{m(p)}\right), m \neq m(p)} \frac{4 \delta \mu_{m(p)}}{3 \delta \mu_{m}} \geqslant 1, \quad \lambda \in S\left(\mu_{m(p)}, 5 \delta \mu_{m(p)}\right) .
\end{aligned}
$$

Следовательно,

$$
\begin{aligned}
\left|g_{p}(z)\right| & =\left|\frac{1}{2 \pi i} \int_{S\left(\mu_{m(p)}, 5 \delta \mu_{m(p)}\right)} \frac{\exp (\lambda z) d \lambda}{a_{p}\left(\lambda-\mu_{m(p)}\right) q_{\tilde{\Lambda}}^{m(p)}(\lambda, \delta)}\right| \\
& \leqslant \sup _{\lambda \in S\left(\mu_{m(p)}, 5 \delta \mu_{m(p)}\right)}|\exp (\lambda z)| \leqslant \exp \left(\operatorname{Re}\left(\mu_{m(p)} z\right)+5 \delta \mu_{m(p)}|z|\right), \quad z \in \mathbb{C} .
\end{aligned}
$$

Покажем, что ряд (5) сходится на множестве $D=\{z: \operatorname{Re} z<\tau \delta\} \cap B(0,4 \tau / 5)$. Пусть $z \in D$. Из последней оценки с учетом определения коэффициентов $c_{p}$ и (7) получаем

$$
|g(z)| \leqslant \sum_{p=1}^{\infty} \exp \left(\operatorname{Re}\left(\mu_{m(p)} z\right)+5 \delta \mu_{m(p)}|z|-6 \tau \delta \mu_{m(p)}\right) \leqslant \sum_{p=1}^{\infty} \exp \left(-\tau \delta \mu_{m(p)}\right)<\infty .
$$

Таким образом, ряд (5) сходится равномерно на $D$. Его члены являются целыми функциями. Следовательно, $g(z)$ - функция, аналитическая в области $D$. Отметим, что сказанное верно при любом выборе чисел $a_{p} \geqslant 1, p=1,2, \ldots$. Покажем теперь, что при подходящем выборе этих чисел функция $g(z)$ разлагается в ряд Дирихле, прямая сходимости которого совпадает с мнимой осью. Используя вычеты, для каждого $p=1,2, \ldots$ получаем

$$
g_{p}(z)=a_{p}^{-1}\left(b_{m(p)} \exp \left(\mu_{m(p)} z\right)+\sum_{\mu_{m} \in \Lambda_{p}, m \neq m(p)} b_{m} \exp \left(\mu_{m} z\right)\right),
$$

где $b_{m(p)}=\left(q_{\tilde{\Lambda}}^{m(p)}\left(\mu_{m(p)}, \delta\right)\right)^{-1}$. Пусть $p=1,2, \ldots$ Для каждого номера $m$, такого, что $\mu_{m} \in \Lambda_{p}$, положим $d_{m}=c_{p} b_{m}\left(a_{p}\right)^{-1}$. В силу (9) и определения чисел $c_{p}$ верно неравенство

$$
\max _{m: \mu_{m} \in \Lambda_{p}} \frac{\ln \left|c_{p} b_{m}\right|}{\mu_{m(p)}} \geqslant \frac{-6 \tau \delta \mu_{m(p)}-\ln \left(q_{\tilde{\Lambda}}^{m(p)}\left(\mu_{m(p)}, \delta\right)\right)}{\mu_{m(p)}} \geqslant 0 .
$$


Поэтому найдется число $a_{p} \geqslant 1$, при котором

$$
\max _{m: \mu_{m} \in \Lambda_{p}} \frac{\ln \left|d_{m}\right|}{\mu_{m}(p)}=0 .
$$

Рассмотрим ряд Дирихле

$$
\sum_{m=1}^{\infty} d_{m} \exp \left(\mu_{m} z\right)
$$

Так как верхняя плотность $\bar{\tau}(\tilde{\Lambda})$ конечна, ясно, что величина $\overline{\lim }_{m \rightarrow \infty} \ln m / \mu_{m}$ равна нулю. Поэтому (см., например, [1, гл. II, §1, теорема 2.1.2], [19]) для ряда (12) имеет место формула Коши-Адамара

$$
\gamma=\varlimsup_{m \rightarrow \infty} \frac{\ln \left|d_{m}\right|}{\mu_{m}}
$$

где $\gamma$ - абсцисса сходимости этого ряда. При этом в полуплоскости $\{z \in \mathbb{C}:$ $\operatorname{Re} z<\gamma\}$ ряд (12) сходится абсолютно (см., например, [1, гл. II, §1, следствие из теоремы 2.1.1]). В силу (11)

$$
\gamma=\varlimsup_{m \rightarrow \infty} \frac{\ln \left|d_{m}\right|}{\mu_{m}}=\varlimsup_{p \rightarrow \infty} \max _{m: \mu_{m} \in \Lambda_{p}} \frac{\ln \left|d_{m}\right|}{\mu_{m}}=0 .
$$

Таким образом, мнимая ось является прямой сходимости ряда (12). Так как он сходится абсолютно в левой полуплоскости, то суммы рядов (5) и (12) совпадают в пересечении $\{z \in \mathbb{C}: \operatorname{Re} z<0\} \cap D$. Это означает, что сумма ряда $(12)$ аналитически продолжается в область $D$, которая содержит отрезок прямой сходимости $[-i \tau 2, i \tau 2]$ длины $\tau$. Лемма доказана.

Пусть $\Lambda=\left\{\lambda_{k}\right\}_{k=1}^{\infty}, D-$ выпуклая область и $H(D)$ - пространство функций, аналитических в $D$, с топологией равномерной сходимости на компактах. Через $W(\Lambda, D)$ обозначим замыкание в $H(D)$ линейной оболочки системы функций $\mathscr{E}=\left\{\exp \left(\lambda_{k} z\right)\right\}_{k=1}^{\infty}$. Тогда $W(\Lambda, D)$ - замкнутое подпространство в $H(D)$, инвариантное относительно оператора дифференцирования. По построению оно допускает спектральный синтез, т. е. система $\mathscr{E}$ полна в $W(\Lambda, D)$. Подпространство $W(\Lambda, D)$ называется нетривиальным, если оно отлично от $H(D)$, т. е. система $\mathscr{E}$ не полна в $H(D)$. По теореме Хана-Банаха нетривиальность подпространства $W(\Lambda, D)$ равносильна существованию ненулевого линейного непрерывного функционала $\nu \in H^{*}(D)$, который аннулирует $W(\Lambda, D)$ или, что эквивалентно, обращается в нуль на элементах системы $\mathscr{E}$. Преобразование Лапласа $f(\lambda)=\nu(\exp (\lambda z))$ устанавливает изоморфизм (см., например, [20, гл. III, §12, теорема 12.3]) между сопряженным пространством $H^{*}(D)$ и пространством $P_{D}$, состоящим из целых функций экспоненциального типа, сопряженные диаграммы которых лежат в области $D$. Таким образом нетривиальность подпространства $W(\Lambda, D)$ равносильна существованию функции $f \in P_{D}$, которая обращается в нуль в точках $\lambda_{k}, k=1,2, \ldots$

Говорят, что в подпространстве $W(\Lambda, D)$ имеет место фундаментальный принцип, если каждый его элемент разлагается в ряд по системе $\mathscr{E}$, сходящийся в топологии пространства $H(D)$. Последнее, согласно теореме Абеля для рядов экспонент (см. [20]), равносильно тому, что этот ряд сходится в каждой точке области $D$. Проблема фундаментального принципа (т. е. выяснение условий, 
при которых он имеет место) для произвольных замкнутых инвариантных подпространств, допускающих спектральный синтез, решена в работах [15], [16]. Следующий результат адаптирует это решение к нашему случаю.

Лемма 4. Пусть $D$ - ограниченная выпуклая область в $\mathbb{C}$, a $\Lambda=\left\{\lambda_{k}\right\}_{k=1}^{\infty}$ - неограниченная строго возрастающая последовательность положительных чисел с конечной максимальной плотностъю $\tau_{0}(\Lambda)$. Предположим, что подпространство $W(\Lambda, D)$ нетривиально и пересечения опорных прямых $\{z \in \mathbb{C}:$ $\left.\operatorname{Re} z=H_{D}(1)\right\} u\left\{z \in \mathbb{C}: \operatorname{Re} z=H_{D}(-1)\right\}$ с границей области $D$ содержсат отрезки длины $2 \pi \tau_{0}(\Lambda)$. Тогда в $W(\Lambda, D)$ имеет место фундаментальный принu,un.

Доказательство. Согласно утверждению 7 теоремы 5.2 в работе [15], в подпространстве $W(\Lambda, D)$ имеет место фундаментальный принцип, если существует целая функция $f$ экспоненциального типа, которая обращается в нуль во всех точках $\lambda_{k}$ и для которой выполнены равенства $\underline{h}_{f}(\lambda)=h_{f}(\lambda)=H_{D}(\lambda), \lambda \in \mathbb{C}$. Последние равносильны тому, что функция $f$ имеет регулярный рост, а ее сопряженная диаграмма совпадает с замыканием $\bar{D}$ области $D$. Для построения такой функции нам необходимо, прежде всего, представить $\bar{D}$ в виде суммы двух выпуклых множеств. Пусть $K$ - отрезок мнимой оси $\left[-\pi \tau_{0}(\Lambda), \pi \tau_{0}(\Lambda)\right]$. Положим

$$
\widetilde{D}=\left\{z \in \mathbb{C}: \operatorname{Re}(z \lambda) \leqslant H_{D}(\lambda)-H_{K}(\lambda), \lambda \in S(0,1)\right\} .
$$

Поскольку множество $\widetilde{D}$ является пересечением полуплоскостей, то оно выпукло. Его опорная функция $H_{\widetilde{D}}(\lambda)$ удовлетворяет неравенству (учитываем положительную однородность)

$$
H_{\widetilde{D}}(\lambda)=\sup _{z \in \widetilde{D}} \operatorname{Re}(\lambda z) \leqslant H_{D}(\lambda)-H_{K}(\lambda), \quad \lambda \in \mathbb{C} .
$$

Отсюда получаем, что $H_{\widetilde{D}}(\lambda)+H_{K}(\lambda) \leqslant H_{D}(\lambda), \lambda \in \mathbb{C}$. Следовательно, $\widetilde{D}+$ $K \subseteq \bar{D}$. Покажем, что имеет место и обратное вложение. Пусть $w \in \bar{D}$. Тогда $w$ принадлежит вертикальному отрезку $\widetilde{K}$ длины $2 \pi \tau_{0}(\Lambda)$ (вообще говоря, не единственному), который целиком содержится в замыкании $\bar{D}$. Этот отрезок лежит на пересечении прямой $\{z \in \mathbb{C}: \operatorname{Re} z=\operatorname{Re} w\}$ с выпуклой оболочкой точки $w$ и параллелограмма, две стороны которого составляют отрезки длины $2 \pi \tau_{0}(\Lambda)$ из условия леммы. Пусть $\tilde{z}-$ середина отрезка $\widetilde{K}$. Тогда $\widetilde{K}=\tilde{z}+K$. Так как $\widetilde{K} \subseteq \bar{D}$, то $\operatorname{Re}(\tilde{z} \lambda)+H_{K}(\lambda)=H_{\widetilde{K}}(\lambda) \leqslant H_{\bar{D}}(\lambda), \lambda \in \mathbb{C}$. Отсюда следует, что $\tilde{z} \in \widetilde{D}$. Поэтому отрезок $\widetilde{K}$, а вместе с ним и точка $w$, содержатся в сумме множеств $\widetilde{D}+K$. Поскольку $w \in \bar{D}$ выбиралась произвольно, то получаем вложение $\bar{D} \subseteq \widetilde{D}+K$. Таким образом, $\bar{D}=\widetilde{D}+K$, т. е. $H_{\widetilde{D}}(\lambda)+H_{K}(\lambda)=$ $H_{\bar{D}}(\lambda)=H_{D}(\lambda), \lambda \in \mathbb{C}$.

Пусть $f_{1}$ - целая функция экспоненциального типа, построенная в лемме 1 для последовательности $\Lambda$. Она имеет регулярный рост, обращается в нуль во всех точках $\lambda_{k}$, а ее сопряженная диаграмма совпадает с отрезком $K$. Тогда $h_{f_{1}}(\lambda)=H_{K}(\lambda), \lambda \in \mathbb{C}$. Через $f_{2}$ обозначим целую функцию экспоненциального типа и регулярного роста с индикатором $h_{f_{2}}$, равным $H_{\widetilde{D}}$ (по поводу ее построения см., например, [1, гл. I, §3, теорема 1.3.2]). Положим $f=f_{1} f_{2}$. Очевидно, что функция $f$ является целой функцией экспоненциального типа и 
имеет регулярный рост. По теореме о сложении индикаторов (см. [16, гл. III, §5, теорема 5])

$$
h_{f}(\lambda)=h_{f_{1}}(\lambda)+h_{f_{2}}(\lambda)=H_{K}(\lambda)+H_{\widetilde{D}}(\lambda)=H_{D}(\lambda), \quad \lambda \in \mathbb{C} .
$$

Таким образом, $f$ - искомая функция. Лемма доказана.

Последний результат этого параграфа частично обращает лемму 4.

Лемма 5. Пусть $D$ - ограниченная выпуклая область в $\mathbb{C}$, a $\Lambda=\left\{\lambda_{k}\right\}_{k=1}^{\infty}$ - неограниченная строго возрастающая последовательность положительных чисел с конечной максимальной плотностью $\tau_{0}(\Lambda)$, такая, что подпространство $W(\Lambda, D)$ нетривиально. Предположим, что в $W(\Lambda, D)$ имеет место фундаментальный приниип. Тогда пересечение опорной прямой $\{z \in \mathbb{C}: \operatorname{Re} z=$ $\left.H_{D}(1)\right\}$ с гранищей области $D$ содержит отрезок длинь $2 \pi \tau_{0}(\Lambda)$.

Доказательство. Если выполнены условия леммы, то, согласно теореме 5.2 из работы [15], существует целая функция экспоненциального типа $f$, обращающаяся в нуль во всех точках $\lambda_{k}$ и такая, что

$$
\underline{h}_{f}(\lambda)=h_{f}(\lambda)=H_{D}(\lambda), \quad \lambda \in \mathbb{C} .
$$

Это означает, что функция $f$ имеет регулярный рост, а ее сопряженная диаграмма совпадает с замыканием области $D$. Тогда, согласно (4), для всех, за исключением не более чем счетного числа, значений $\varphi$ верно равенство

$$
\tau(-\varphi, \varphi, \tilde{\Lambda})=\frac{1}{2 \pi} s(-\varphi, \varphi, D)
$$

где $\tilde{\Lambda}$ - нулевое множество функции $f, s(-\varphi, \varphi, D)$ - длина дуги $\gamma(\varphi)$ границы $\partial D$, заключенной между точками опоры $z(\varphi), z(-\varphi) \in \partial D$ опорных прямых $l( \pm \varphi)=\left\{z: \operatorname{Re}\left(z e^{ \pm \varphi i}\right)=H_{D}\left(e^{ \pm \varphi i}\right)\right\}$ соответственно. Поскольку $\Lambda$ является частью множества $\tilde{\Lambda}$, которое измеримо, то для всех, за исключением не более чем счетного числа, значений $\varphi$ имеем

$$
\begin{aligned}
\tau_{0}(\Lambda) & =\varlimsup_{\delta \rightarrow 0} \varlimsup_{r \rightarrow \infty} \frac{n(r, \Lambda)-n((1-\delta) r, \Lambda)}{\delta r} \\
& \leqslant \varlimsup_{\delta \rightarrow 0} \varlimsup_{r \rightarrow \infty} \frac{n(-\varphi, \varphi, r, \Lambda)-n(-\varphi, \varphi,(1-\delta) r, \Lambda)}{\delta r} \\
& =\varlimsup_{\delta \rightarrow 0}\left(\lim _{r \rightarrow \infty} \frac{n(-\varphi, \varphi, r, \Lambda)}{\delta r}-\lim _{r \rightarrow \infty} \frac{n(-\varphi, \varphi,(1-\delta) r, \Lambda)}{\delta r}\right) \\
& =\varlimsup_{\delta \rightarrow 0}\left(\frac{\tau(-\varphi, \varphi, \tilde{\Lambda})}{\delta}-\frac{(1-\delta) \tau(-\varphi, \varphi, \tilde{\Lambda})}{\delta}\right)=\tau(-\varphi, \varphi, \tilde{\Lambda}) .
\end{aligned}
$$

Когда $\varphi \rightarrow 0$, дуга $\gamma(\varphi)$ стягивается к множеству $\partial D \cap\left\{z: \operatorname{Re} z=H_{D}(1)\right\}$, которое представляет собой вертикальный отрезок (возможно, вырождающийся в точку). Из (13) и (14) следует, что его длина не меньше, чем $2 \pi \tau_{0}(\Lambda)$. Лемма доказана.

\section{§3. Доказательство теоремы 1}

Сначала докажем импликацию $2 \Longrightarrow 1$. Пусть $K^{\prime}$ - отрезок длины $2 \pi \tau_{0}$ на прямой сходимости $\{z: \operatorname{Re} z=\gamma\}$ ряда $(1)$, сумму которого обозначим через $g$. Предположим, что на $K^{\prime}$ функция $g$ не имеет особых точек. Другими словами, 
для каждой точки $w \in K^{\prime}$ функция $g$ аналитически продолжается в некоторый круг $B(w, r(w))$. Если круги $B\left(w_{1}, r\left(w_{1}\right)\right)$ и $B\left(w_{2}, r\left(w_{2}\right)\right)$ пересекаются, то непустым будет также пересечение $B\left(w_{1}, r\left(w_{1}\right)\right) \cap B\left(w_{2}, r\left(w_{2}\right)\right) \cap\{z: \operatorname{Re} z<\gamma\}$. Следовательно, по теореме единственности продолжения функции $g$ в круги $B\left(w_{1}, r\left(w_{1}\right)\right)$ и $B\left(w_{2}, r\left(w_{2}\right)\right)$ совпадают во всех точках их пересечения. Таким образом, функция $g$ аналитически продолжается в некоторый открытый прямоугольник $Q$, содержащий $K^{\prime}$, со сторонами, параллельными осям координат.

Пересечение $Q \cap\{z: \operatorname{Re} z<\gamma\}$ содержит некоторый сдвиг $K+z_{0}$ отрезка мнимой оси $K=\left[-i \pi \tau_{0}(\Lambda), i \pi \tau_{0}(\Lambda)\right]$. Кроме того, прямоугольник $Q$ можно представить в виде суммы $Q^{\prime}+K$, где $Q^{\prime}$ также является открытым прямоугольником. Пусть $f$ - функция, существование которой утверждается в лемме 1. Так как $f$ обращается в нуль во всех точках $\lambda_{k}$, а функция $g$ аппроксимируется равномерно на компактах линейными комбинациями элементов системы $\mathscr{E}$ в некоторой окрестности сдвига компакта $K$ (она представляется рядом (1) в указанном пересечении), то, согласно теореме 12.1 из работы [21], эта аппроксимация продолжается в область $Q=Q^{\prime}+K$. Таким образом, $g \in W(\Lambda, Q)$. Функция $f(\lambda) \exp \left(\lambda z_{0}\right)$, сопряженной диаграммой которой является компакт $K+z_{0}$, принадлежит пространству $P_{Q}$. Это, как отмечалось выше, означает, что $W(\Lambda, Q)$ нетривиально. Тогда по лемме 4 функция $g$ представляется рядом (1) в прямоугольнике $Q$, а значит, и в полуплоскости, содержащей $Q$. Поскольку представление рядом Дирихле всегда является единственным, то это противоречит тому, что $\gamma$ - абсцисса сходимости ряда (1) с суммой $g$. Следовательно, наше предположение неверно, т. е. функция $g$ имеет хотя бы одну особую точку на отрезке $K^{\prime}$.

Докажем теперь обратное утверждение: $1 \Longrightarrow 2$. Предположим, что $\widetilde{S}_{\Lambda}=$ $-\infty$. Тогда по лемме 3 существует ряд вида (1), сумма которого аналитически продолжается через некоторый отрезок длины $2 \pi \tau$, лежащий на прямой сходимости этого ряда. Это противоречит утверждению 1. Следовательно, величина $\widetilde{S}_{\Lambda}$ конечна. Тогда, как отмечалось выше, $S_{\Lambda}=0$. Кроме того, по лемме 2 максимальная плотность $\tau_{0}(\Lambda)$ конечна. Предположим, что $\tau_{0}(\Lambda)>\tau$, и выберем $\varepsilon>0$, удовлетворяющее условию $\tau_{0}(\Lambda)-\varepsilon \geqslant \tau$. Пусть $D^{\prime}$ - область, ограниченная вертикальными прямыми $\{z \in \mathbb{C}: \operatorname{Re} z=1\},\{z \in \mathbb{C}: \operatorname{Re} z=-1\}$ и прямыми, проходящими через точки с координатами $\left(0, i \pi \tau_{0}(\Lambda)\right),\left(1, i \pi\left(\tau_{0}(\Lambda)-\varepsilon\right)\right)$ и $\left(0,-i \pi \tau_{0}(\Lambda)\right),\left(1,-i \pi\left(\tau_{0}(\Lambda)-\varepsilon\right)\right)$. Тогда $D^{\prime}-$ равнобедренная трапеция, одно основание которой лежит на прямой $\{z \in \mathbb{C}: \operatorname{Re} z=-1\}$ и имеет длину, строго большую, чем $2 \pi \tau_{0}(\Lambda)$, а другое лежит на прямой $\{z \in \mathbb{C}: \operatorname{Re} z=1\}$ и имеет длину, равную $2 \pi\left(\tau_{0}(\Lambda)-\varepsilon\right)$.

Подпространство $W\left(\Lambda, D^{\prime}\right)$ нетривиально по тем же соображениям, что и выше. Пересечение опорной прямой $\left\{z \in \mathbb{C}: \operatorname{Re} z=H_{D^{\prime}}(1)\right\}$ с границей области $D^{\prime}$ не содержит отрезка длины $2 \pi \tau_{0}(\Lambda)$ (оно является отрезком длины $2 \pi\left(\tau_{0}(\Lambda)-\varepsilon\right)$. Следовательно, согласно лемме 5, существует функция $g \in W\left(\Lambda, D^{\prime}\right)$, которая не представляется рядом (1) в области $D^{\prime}$.

Рассмотрим область $D^{\prime \prime}=D^{\prime} \cap\{z \in \mathbb{C}: \operatorname{Re} z<0\}$. Она является равнобедренной трапецией, одно основание которой лежит на прямой $\{z \in \mathbb{C}: \operatorname{Re} z=-1\}$ и совпадает с соответствующим основанием трапеции $D^{\prime}$, а другое лежит на мнимой оси и совпадает с отрезком $\left[-i \pi \tau_{0}(\Lambda), i \pi \tau_{0}(\Lambda)\right]$. Таким образом, пересечения опорных прямых $\left\{z \in \mathbb{C}: \operatorname{Re} z=H_{D^{\prime \prime}}(-1)=-1\right\}$ и $\{z \in \mathbb{C}: \operatorname{Re} z=$ $\left.H_{D^{\prime}}(1)=0\right\}$ с границей области $D^{\prime \prime}$ содержат отрезки длины $2 \pi \tau_{0}(\Lambda)$. Как и 
$W\left(\Lambda, D^{\prime}\right)$, подпространство $W\left(\Lambda, D^{\prime \prime}\right)$ нетривиально. Тогда по лемме 4 каждая функция из $W\left(\Lambda, D^{\prime \prime}\right)$ представляется в области $D^{\prime \prime}$ рядом Дирихле (1). Поскольку $D^{\prime \prime} \subset D^{\prime}$, то $W\left(\Lambda, D^{\prime \prime}\right)$ содержит $W\left(\Lambda, D^{\prime}\right)$. Следовательно, функция $g$ в области $D^{\prime \prime}$ разлагается в ряд Дирихле. Последний же всегда сходится в некоторой полуплоскости $\{z \in \mathbb{C}: \operatorname{Re} z<\gamma\}$, а в дополнительной полуплоскости $\{z \in \mathbb{C}: \operatorname{Re} z>\gamma\}$ расходится. Это означает, что $D^{\prime \prime} \subset\{z \in \mathbb{C}: \operatorname{Re} z<\gamma\}$, т. е. $\gamma>0$. Кроме того, также $\gamma<1$. Действительно, в противном случае полуплоскость сходимости $\{z \in \mathbb{C}: \operatorname{Re} z<\gamma\}$ будет содержать полуплоскость $G=\left\{z \in \mathbb{C}: \operatorname{Re} z<H_{D^{\prime}}(1)=1\right\}$. Следовательно, функция $g$ будет представляться рядом (1) в области $D^{\prime} \subset G$. Это противоречит выбору функции $g$.

По построению $g$ аналитична в области $D^{\prime}$. Она пересекает прямую сходимости $\{z \in \mathbb{C}: \operatorname{Re} z=\gamma\}$ по интервалу, длина которого строго больше $2 \pi\left(\tau_{0}(\Lambda)-\varepsilon\right)$. Таким образом, мы получили сумму ряда (1), не являющуюся целой функцией и не имеющую особых точек на некотором отрезке прямой сходимости, длина которого строго больше $2 \pi \tau$. Это противоречит утверждению 1. Следовательно, наше предположение $\tau_{0}(\Lambda)>\tau$ неверно. Теорема доказана.

Замечание. Частными случаями теоремы 1 являются все отмеченные в начале работы результаты. Выше было показано, что в случае, когда $\lambda_{k+1}-\lambda_{k} \geqslant$ $h>0, k=1,2, \ldots$, величина $\widetilde{S}_{\Lambda}$ конечна, а потому $S_{\Lambda}=0$. Таким образом, если максимальная плотность $\tau_{0}(\Lambda)$ (а вместе с ней и плотность) равна нулю, то из теоремы 1 сразу вытекают результаты Адамара и Фабри для рядов Тейлора, а также результаты Пойи, Карлсона и Ландау для рядов Дирихле. Если $\tau_{0}(\Lambda) \geqslant 0$, то из теоремы 1 получаем результаты Пойи, Фукса и Мальявена для рядов Тейлора. Наконец, если индекс конденсации Бернштейна $\gamma(\Lambda)$ равен нулю, то, как показано в работе [14] (следствие 4.2), равна нулю и величина $S_{\Lambda}$. Следовательно, из теоремы 1 вытекает теорема Бернштейна.

Отметим, что в случае, когда последовательность $\Lambda$ имеет плотность, величина $S_{\Lambda}$ в теореме 1 может быть заменена на $\gamma(\Lambda)$. Это вытекает из теоремы Бернштейна и существования специальной функции, о которой упоминалось во введении. В этом случае величины $S_{\Lambda}$ и $\gamma(\Lambda)$ равны или не равны нулю одновременно. В общем же случае из равенства нулю величины $S_{\Lambda}$ не следует, что $\gamma(\Lambda)=0$. В этой связи рассмотрим следующий пример.

Пусть $\Lambda=\bigcup_{m=1}^{\infty} \Lambda_{m}, \Lambda_{m}=\left\{\lambda_{p(m)}, \lambda_{p(m)+1}, \ldots, \lambda_{\tilde{p}(m)}\right\}$, где $\tilde{p}(m)=p(m)+$ $\lambda_{p(m)} / 2, \lambda_{p+1}=\lambda_{p}+1, p=p(m), \ldots, \tilde{p}(m)-1$, и $\lambda_{p(m)}$ - четные положительные числа, которые мы сейчас определим. Фиксируем $\varepsilon>0$. Положим $\lambda_{p(1)}=\lambda_{1}=2$ и для каждого $m \geqslant 2$ в качестве $\lambda_{p(m)}$ возьмем какое-нибудь из указанных чисел, для которого $\lambda_{p(m)}>\lambda_{\tilde{p}(m-1)}+1$ и выполнено также неравенство

$$
\prod_{p=1}^{\tilde{p}(m-1)}\left|1-\frac{\lambda_{p(m)}^{2}}{\lambda_{p}^{2}}\right|<\exp \left(\varepsilon \lambda_{p(m)}\right) .
$$

Поскольку $\lambda_{p+1}-\lambda_{p} \geqslant 1, p=1,2, \ldots$, то, как и выше, $S_{\Lambda}=0$. С другой стороны,

$$
\left|L^{\prime}\left(\lambda_{p(m)}\right)\right|=\frac{2}{\lambda_{p(m)}} \prod_{p \neq p(m)}\left|1-\frac{\lambda_{p(m)}^{2}}{\lambda_{p}^{2}}\right| .
$$


Следовательно,

$$
\begin{aligned}
\ln \left|L^{\prime}\left(\lambda_{p(m)}\right)\right| & \leqslant \varepsilon \lambda_{p(m)}+\sum_{p=p(m)+1}^{\tilde{p}(m)} \ln \left(1-\frac{\lambda_{p(m)}^{2}}{\lambda_{p}^{2}}\right)+\sum_{p=p(m+1)}^{\infty} \ln \left(1-\frac{\lambda_{p(m)}^{2}}{\lambda_{p}^{2}}\right) \\
& <\varepsilon \lambda_{p(m)}+\sum_{p=p(m)+1}^{\tilde{p}(m)} \ln \left(1-\frac{\lambda_{p(m)}^{2}}{\lambda_{p}^{2}}\right) \\
& <\varepsilon \lambda_{p(m)}+\sum_{p=p(m)+1}^{\tilde{p}(m)} \ln \left(2 \frac{p-p(m)}{\lambda_{p(m)}}\right) \\
& =\varepsilon \lambda_{p(m)}+(\tilde{p}(m)-p(m)) \ln \frac{2}{\lambda_{p(m)}}+\ln (\tilde{p}(m)-p(m)) ! \\
& <1+\varepsilon \lambda_{p(m)}+(\tilde{p}(m)-p(m))\left(\ln \frac{2}{\lambda_{p(m)}}+\ln \frac{\tilde{p}(m)-p(m)}{2}\right) \\
& =1+\varepsilon \lambda_{p(m)}-\frac{\lambda_{p(m)}}{2} \ln 2 .
\end{aligned}
$$

Отсюда при достаточно малом $\varepsilon>0$ получаем, что $\gamma(\Lambda)>0$. Этот пример показывает, что в общем случае в теореме 1 величину $S_{\Lambda}$ нельзя заменить на $\gamma(\Lambda)$.

\section{ЛитеРАТУРА}

[1] А. Ф. Леонтьев, Рядъ экспонент, Наука, М., 1976.

[2] J. Hadamard, Essai sur l'etude des fonctions donnes par leur développement de Taylor, J. Math. Pures Appl. Ser. (4), 4(8) (1892), 101-106.

[3] E. Fabry, Sur les points singuliers d'une function donnée par son développement en série et l'impossibilité du prolongement analytique dans des cas très généraux, Ann. Ecole Norm. Sup. (3), 13 (1896), 367-399.

[4] P. Koosis, The Logarithmic Integral, vol. 1, Cambridge University Press, Cambridge, 1997.

[5] G. Pólya, Untersuchungen über Lücken und Singularitäten von Potenzreihen, Math. Z., 29:1 (1929), 549-640.

[6] Л. Бибербах, Аналитическое продолжение, Наука, М., 1967.

[7] W. Fuchs, On the growth of functions of mean type, in: Proc. Edinburgh Math. Soc., Ser. 2, vol. 9, 1954, 53-70.

[8] P. Malliavin, Sur la croissance radiale d'une function méromorphe, Illinois J. Math., 1 (1957), 259-296.

[9] P. Koosis, The Logarithmic Integral, vol. 2, Cambridge University Press, Cambridge, 2008.

[10] G. Pólya, Über die Existenz unendlich vieler singulärer Punkte auf der Konvergenzgeraden gewisser Dirichletscher Reihen, Sitzungber. Preuss. Akad. Wiss., Phys.-Math. Kl. (1923), 45-50.

[11] G. Pólya, Eine Verallgemeinerung des Fabryschen Lückensatzes, Nachr. Ges. Wiss. Gottingen, Math.-Phys. Kl. (1927), 187-195.

[12] V. Bernstein, Leçons sur les progrèss récents de la théorie des séries de Dirichlet, Gauthier-Villars, Paris, 1933.

[13] А. Ф. Леонтьев, Целье функиии. Рлды экспонент, М., Наука, 1983. 
[14] О. А. Кривошеева, Особъе точки суммъ ряда экспоненциалъных мономов на границе области сходимости, Алгебра и анализ, 23:2 (2011), 162-205.

[15] А. С. Кривошеев, Фундаментальный приниип для инвариантных подпространств в выпуклых областях, Изв. РАН, сер. матем., 68:2 (2004), 71-136.

[16] О. А. Кривошеева, А. С. Кривошеев, Критерий справедливости фундаментального приниипа для инвариантных подпространств в ограниченных выпуклых областях комплексной плоскости, Функц. анализ и его прил., 46:4 (2012), 14-30.

[17] Б. Я. Левин, Распределение корней цельх функиий, Гостехиздат, М., 1956.

[18] П. Лелон, Л. Груман, Целье функиии многих комплексных переменных, Мир, М., 1989.

[19] О. А. Кривошеева, Область сходимости рядов экспоненииальньх мономов, Уфимск. матем. ж., 3:2 (2011), 43-56.

[20] В. В. Напалков, Уравнения свертки в многомерных пространствах, Наука, М., 1982.

[21] И. Ф. Красичков-Терновский, Инвариантные подпространства аналитических функций. III: О распространении спектралъного синтеза, Матем. сб., 88(130):3 (1972), 331-352.

Башкирский государственный университет e-mail: kriolesya2006@yandex.ru

Поступила в редакцию Институт математики с ВЦ УНЦ РАН

8 мая 2013 г.

e-mail: shaig@anrb.ru 